\title{
To Approach or to Avoid? Motivation Differentially Mediates the Effect of Hardiness on Depressive Symptoms in Chinese Military Personnel
}

\author{
Xiaoxia Wang $\mathbb{D},{ }^{1}$ Janet Yuen-Ha Wong $\mathbb{D},{ }^{2}$ Linkun Zhai, ${ }^{3}$ \\ Ruicheng Wu, ${ }^{3}$ Tianhao Huang $\left(\mathbb{D},{ }^{3}\right.$ Renqiang $\mathrm{He},{ }^{3}$ Yang Xiao, ${ }^{3}$ \\ Yang Yu, ${ }^{3}$ Xiangji Kong, ${ }^{3}$ Xiaoyan Zhou $\left(\mathbb{D},{ }^{4}\right.$ and Hui Yang $(\mathbb{D})^{4}$ \\ ${ }^{1}$ Department of Basic Psychology, College of Psychology, Army Medical University, Chongqing 400038, China \\ ${ }^{2}$ School of Nursing, LKS Faculty of Medicine, The University of Hong Kong, Hong Kong \\ ${ }^{3}$ Cadet Brigade, Army Medical University, Chongqing 400038, China \\ ${ }^{4}$ Department of Clinical Psychology, The Mental Health Center of Chongqing, Chongqing 500000, China
}

Correspondence should be addressed to Xiaoyan Zhou; 269402743@qq.com and Hui Yang; yanghui0925@126.com

Received 6 November 2018; Accepted 18 April 2019; Published 27 May 2019

Guest Editor: Gabriela Topa

Copyright (c) 2019 Xiaoxia Wang et al. This is an open access article distributed under the Creative Commons Attribution License, which permits unrestricted use, distribution, and reproduction in any medium, provided the original work is properly cited.

Objective. To investigate the mediation effect of approach/avoidance motivation between hardiness and depressive symptoms. Methods. Cross-sectional design was utilized. Two independent samples of military servicemen (G1: military personnel in the Armed Forces; G2: Chinese army military cadets) $(\mathrm{n} 1=98, \mathrm{n} 2=140)$ were sampled and investigated. The assessment tools of hardiness scale (DRS), behavioral activation and inhibition scales (BAS/BIS), and Center for Epidemiological Survey-Depression Scale (CES-D)/Beck Depression Inventory (BDI) were used. General linear model was conducted to examine the predictive role of hardiness (DRS) and motivation (BAS/BIS) on depressive symptoms (CES-D or BDI). The mediating role of BAS/BIS between hardiness and depressive symptoms was examined. Results. (1) Across army soldiers and military medical university cadets, hardiness $(\beta=-0.394, P<0.001)$ and behavioral inhibition $(\beta=0.297, P<0.001)$ significantly predicted depressive symptoms. (2) For soldiers only, behavioral inhibition mediated the significant association between hardiness and depressive symptoms $(\beta=-0.043$, $\mathrm{SE}=0.027,95 \% \mathrm{CI}=-0.130 \sim-0.008)$. (3) For cadets only, behavioral activation-Drive significantly predicted depressive symptoms $(\beta=-$ $0.237, P=0.012)$, and hardiness operates through behavioral activation-Drive to influence depressive symptoms $(\beta=-0.057, \mathrm{SE}=0.036$, $95 \% \mathrm{CI}=-0.151 \sim-0.078)$. Conclusion. Individuals who are low in hardiness and behavioral activation-Drive and who are high in behavioral inhibition showed more severe depressive symptoms. The relationship between hardiness and depressive symptoms was mediated by behavioral activation-Drive in cadets and behavioral inhibition in soldiers. The proposed model offers a useful approach for the development of hardiness training programs to alter approach/avoidance motivation in the military context. Future training program of hardiness could lay more emphasis on promotion of perseverance in pursuing goals in hardy individuals, which may in turn improve active coping.

\section{Introduction}

Conceptually, hardiness is deeply rooted in existentialism which emphasizes hardiness as commitment to challenges and motivation to cope with stressful circumstances to achieve meaning of life [1]. In the past few decades, an extensive body of researches on hardiness has been conducted in areas of clinical and military psychology [2]. Conceptually, hardiness is an individual disposition or style that remains relatively stable across cultures and could be shaped under training conditions. The construct of hardiness was first proposed by Kobasa [3] and then defined by Maddi as the constellation of three intercorrelated dimensions (3Cs): Commitment (the positive attitude, belief, and behavioral tendency exhibited by individuals who could engage life and work with commitment rather than retreating into isolation), 
Challenge (to see changes in life as challenges to grow and adjust effectively), and Control (to believe that they could exert control over the outcome) [4]. Across a range of occupational contexts and stressful conditions, converging evidence has indicated the buffering effect of hardiness against stressrelated illness [3]. Greater level of psychological hardiness predicted adaptive immune and neuroendocrine responses to stress [5] and high level of happiness [6].

Military personnel are faced with stressful work situations including frequent deployments, family separation, life-threating missions, and long work hours. Hardiness has received attention in military populations for its protective role against stress and maintains a healthy and stable state. For example, in military context which involved high levels of deployment-related stress, hardiness is found to be related to less depression and posttraumatic stress disorder (PTSD) [7]. An important reason why hardy people are more effective in stressful situations is their active coping strategies [8]. Earlier studies showed that adaptive coping (e.g., problem-focused, support-seeking) and maladaptive coping (e.g., avoidant coping) mediated the hardiness-illness relationship [9]. More use of positive coping strategies (e.g., active coping and planning) and less use of negative coping strategies (e.g., behavioral disengagement) were identified in those active service members and veterans with greater hardiness $[10,11]$. Specifically, Commitment enhanced mental health by the use of emotion-focused coping strategies. Control improved mental health by the use of problem-focused and supportseeking strategies [12]. Persons low in hardiness are more likely to use avoidant coping such as substance/alcohol abuse $[13,14]$ on the one hand and benefit from social cohesion and report less mental health problems on the other hand [15].

However, further evidence suggested that coping mediates the relationships between dispositional motivation and psychopathological symptoms [16]. Reinforcement sensitivity theory (RST) defined two motivation systems: (1) behavioral activation system (BAS), which guides approach motivation towards reward, and (2) behavioral inhibition system (BIS), which directs avoidance motivation away from punishment [17]. Furthermore, dispositional motivation is a distal predictor of behavior, while coping strategies may be seen as the proximal predictor of behavior [18]. High BIS sensitivity predisposed the individuals to recruit more attentional resources for detection of potential failure to obtain reward [19] and to adopt more avoidant coping strategies [18]. In contrast, BAS sensitivity played a protective role in working against maladaptive avoidant coping (e.g., gambling and alcohol use) [20]. Depression is characteristic of reduced BAS [21-23] and increased BIS [23, 24], or the combination of both [25]. Presumably, a coping style based on weak motivation to seek reward (lower BAS) and strong motivation to avoid punishment (higher BIS) may lead to increased feelings of depression. Mediational study could provide more insights about the relationship between hardiness and mental health outcome. Therefore, we proposed that approach/avoidance motivation may act as mediators between hardiness and depressive symptoms, which has not been tested directly. The aim of the current study is to (1) confirm the predicting effects of hardiness and BAS/BIS on depressive symptoms and (2) infer the mediating role of BAS/BIS between hardiness and depression symptoms.

\section{Methods}

2.1. Participants. To replicate the results within the relatively small sample, we conducted the investigation on another sample with similar age range. Therefore, two types of military personnel were investigated (G1: military personnel in the Armed Forces; G2: military medical university cadets).

G1. A group of military personnel in the Armed Forces were under survey $(n=101$; all male). The questionnaires with missing values $(n=3)$ were excluded. Ninety-eight valid questionnaires were retained.

G2. A group of military medical university cadets were under survey ( $\mathrm{n}=142$; male: 137 , female: 5$)$. Those questionnaires with missing values $(n=2)$ were excluded, with the remaining 140 valid questionnaires.

2.2. Measurements. The self-reported questionnaire included: (1) items on demographic and background variables, such as age, education, gender, and marital status, and (2) three measurement tools included DRS, BBS, CES-D (sample 1), or BDI (sample 2).

(1) Dispositional Resilience Scale (DRS). DRS was originally developed by Paul Bartone [26] and was translated into Chinese version with satisfactory psychometric properties [27]. The DRS included 15 items and comprised of three dimensions (3Cs): Challenge, Commitment, and Control. The DRS was rated on a 4-point Likert scale ranging from 1 (not at all true) to 4 (completely true). The DRS has acceptable internal consistency (group 1: Cronbach alpha=0.607; group 2: Cronbach alpha=0.662). The 3-week test-retest reliability coefficient was 0.78 [26].

(2) Behavioral Inhibition System and Behavioral Activation System Scale (BIS/BAS Scale, BBS). BBS was a reliable and valid instrument based on Gray's theory of reinforcement sensitivity [28]. The BBS was translated into Chinese and revised by Yanzhang $\mathrm{Li}$ [29]. The translated scale has 18 items and consists of 4 subscales: Behavioral Inhibition System (BIS) subscale (to measure avoidance motivation), Reward Responsiveness (RR), and Drive and the Fun Seeking (FS) subscale (to measure approach motivation). The RR, Drive, and FS subscales are comprised of behavioral activation system (BAS). The RR subscale measures the responsivity to current or anticipated positive stimuli. The Drive subscale measures the persistent pursuit of goals. The FS subscale measures the on-the-moment desire to obtain rewards or approach positive stimuli [28]. The scale uses a 4-point Likert scale ranging from "completely agree" to "completely disagree". The 2-month test-retest reliability was 0.59 0.69 [29]. The DRS has good internal consistency (group 1: Cronbach alpha=0.843; group 2: Cronbach alpha $=0.833$ ).

(3) The Center for Epidemiological Survey-Depression Scale (CES-D). CES-D was developed by the National Institute of 
Mental Health [30]. The CES-D aims to (1) screen for individuals with mild to severe depressive symptoms; (2) assess the severity of depressive symptoms in the past one week. The CES-D is comprised of 20 items which measure nine groups of depressive symptoms (Sadness, Anhedonia, Loss of appetite, Sleep problems, Difficulty in thinking/concentration, Feelings of worthlessness, Fatigue, Agitation, and Suicidal ideation) as defined by the DSM (Diagnostic and Statistical Manual) of APA (American Psychiatric Association). The CES-D is scored with a 4-point Likert scale ranging from 0 ("Not at all or less than one day") to 3 ("5-7 days"). The range of possible scores of CES-D is between 0 (for those who respond 0 to all 20 questions) and 60 (for those who respond 3 to all 20 questions). People who have a total score of $\geq 16$ but do not meet clinical criteria for major depressive episode are deemed as having possible/probable major depressive episode [30]. The CES-D has good internal consistency in the army soldiers (group 1: Cronbach alpha=0.763).

(4) Beck Depression Inventory-II (BDI-II) Developed by Beck in 1967. Similar to CES-D, it can be used to screen depression as well as for the assessment of the severity of depression in patients. It measures three components of depressive symptoms: (1) negative attitude or negative emotions such as pessimism and helplessness; (2) physical symptoms such as fatigue and sleep problems; (3) difficulties in operation with the feeling that work is more difficult than before. The $\mathrm{BDI}$ is scored with a four-point Likert scale ranging from 0 (never/rare) to 3 (very often), with total scores indicating the severity of depressive symptoms (0 13: no depression, 14 19: mild depression, 20 28: moderate depression, and 29 63: severe depression). The BDI-II has good internal consistency in the military medical university cadets (group 2: Cronbach alpha $=0.913$ ).

2.3. Procedure. All procedures were approved by Ethics Committee of Army Medical University (Chongqing, China). A group of military personnel in the Armed Forces (sample 1) and military medical university cadets (sample 2) were administered with paper and pencil questionnaires which assessed hardiness, approach/avoidance motivation and depressive symptoms. Before the test, seven undergraduate investigators were trained to become familiar with the test procedures, instructions and measurement tools. Verbal informed consent of all participants (sample 1) was obtained during July to August in 2015. Written informed consent of all participants (sample 2) was obtained during October of 2016 to May of 2017. Different informed consent forms and depression scales were utilized because two studies were conducted independently during 2015 2017. However, the documentation of the consent process including the names of all participants, information provided, and date consent obtained was kept in the study record. Participants completed the questionnaires in the classroom (sample 1) and laboratories (sample 2) with the same procedures and instructions, under the guidance of one investigator for each participant. This procedure may preclude the possibilities that the measures could be affected by the test condition.
Furthermore, the use of two independent samples may allow us to draw robust conclusions about the effects found.

2.4. Statistical Analysis. Statistical Package for the Social Sciences version 22.0 (SPSS Inc., Chicago, IL) was used as the statistical software. General linear regression analyses were conducted with hardiness as independent variable, BAS, and BIS as mediating variables and depressive symptom (CES-D or BDI scores) as dependent variable. The predictive effect of independent variables (DRS, BAS, and BIS) on depressive symptoms (CES-D or BDI) was tested. PROCESS macro for SPSS [31] (model 4) were used, with hardiness as independent variable, BAS and BIS as mediating variables, and depressive symptoms (CES-D or BDI scores) as dependent variables. The bootstrap sampling method was adopted (resample size $=1000$ ) with $95 \%$ confidence interval to compute the indirect effect of BAS/BIS. The default setup of bootstrap sampling in SPSS macro PROCESS is 1000, which is sufficient for preliminary analyses. The results of mediation analysis were robust when 5000 sampling was utilized, which is the recommended number of sampling for final reporting (Preacher et al., 2008). These variables (hardiness, BAS/BIS, CES-D/BDI) were $\mathrm{z}$-transformed and entered simultaneously into the regression analyses.

Then in order to verify the repeatability of the above results, the two data sets were combined. For convenience of direct comparison, the depressive symptom of the combined data set included standard score of the CES-D or BDI. The scores of other scales (including hardiness, BAS and BIS) are standardized. And then the above analyses were repeated on the assumption that the tested variables conform to the normal distribution.

\section{Results}

Descriptive statistics were listed below for army soldiers (sample 1) and military medical university cadets (sample 2 ). Between-group comparison of hardiness and motivation revealed that cadets had greater levels of hardiness and behavioral activation/inhibition than soldiers (Table 1). Specifically, greater commitment was shown in cadets than soldiers $(\mathrm{t}=-0.28, P=0.006)$, while challenge and control levels were comparable between groups $(\mathrm{t}=-0.83, P=0.41 ; \mathrm{t}=1.08$, $P=0.28)$.

The inferential statistical analyses (i.e., general linear regression and mediating analyses) were performed on the combined sample and then were repeated for sample 1 and 2 , respectively. We explored the mediating role of subfactors of behavioral activation (Reward Responsiveness, Drive, and Fun Seeking), which were entered as mediators between hardiness and depressive symptoms. (1) For soldiers, behavioral inhibition was positively related to depressive symptoms $(\beta=0.261, P=0.029)$, while hardiness negatively predict the depressive symptoms $(\beta=-0.386, P<0.001)$. Behavioral inhibition $(\beta=-0.043, \mathrm{SE}=0.027,95 \% \mathrm{CI}=-0.130 \sim$ -0.008) mediated the significant association between hardiness and depressive symptoms. (2) For cadets, behavioral inhibition was positively related to depressive symptoms $(\beta=0.350, P<0.001)$, while hardiness and behavioral 
TABLE 1: The hardiness personality, avoidance motivation, and depressive symptoms of the military community.

\begin{tabular}{lccc}
\hline & Soldiers & Cadets \\
& $(\mathrm{M} \pm$ S.D. $)$ & $21.30 \pm 1.89$ \\
Age & $15 \sim 30^{\mathrm{a}}$ & $135 / 5$ & $\mathrm{t}(P)$ \\
Gender (male/female) & $98 / 0$ & $0 / 0 / 0 / 140$ & -- \\
Education (middle school/high & $26 / 64 / 7 / 1$ & $43.20 \pm 4.05$ & -- \\
school/junior college/university) & $41.58 \pm 5.81$ & $12.40 \pm 1.84$ & $-2.38 *$ \\
DRS & $11.33 \pm 2.27$ & $13.32 \pm 1.62$ & $-3.85 * * *$ \\
BAS-drive & $12.35 \pm 2.04$ & $15.03 \pm 1.98$ & $-4.07 * * *$ \\
BAS-reward responsiveness & $13.72 \pm 2.69$ & $18.74 \pm 2.95$ & $-4.08 * * *$ \\
BAS-fun seeking & $13.50 \pm 3.25$ & $0.004 \pm 1.00$ & $-12.91 * * *$ \\
BIS & $-0.0007 \pm 1.01$ & -0.04 \\
Z_CES-D/Z_BDI & & - \\
\hline
\end{tabular}

Note: $* P<0.05, * * P<0.01$, and $* * * P<0.001{ }^{a}{ }^{a}$ The age range $(1=15 \sim 20$ years; $2=21 \sim 25$ years; $3=26 \sim 30$ years; $4=31 \sim 35$ years; $5=36$ years and above $)$ was collected. DRS=Dispositional Resilience Scale; BAS=Behavioral Activation Scale; BIS=Behavioral Inhibition Scale; Z_CES-D=Z value of CES-D (Center for Epidemiological Survey Depression Scale) total score; Z_BDI=Z value of BDI (Beck Depression Inventory) total score.

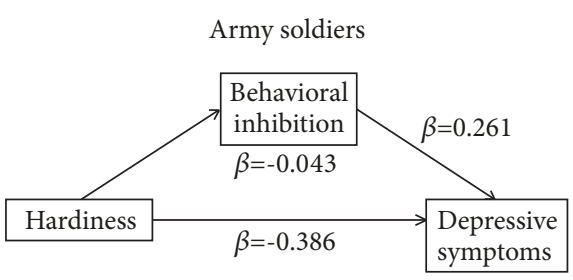

(a)

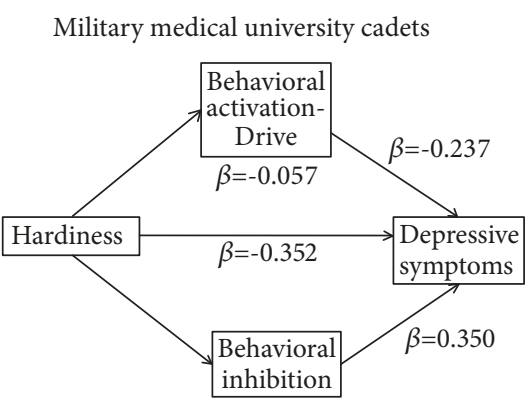

(b)

FIGURE 1: The mediating role of motivation between hardiness and depressive symptoms in military personnel.

activation-Drive negatively predict the depressive symptoms $(\beta=-0.352, P<0.001 ; \beta=-0.237, P=0.012)$. Behavioral activation-Drive $(\beta=-0.057, \mathrm{SE}=0.036,95 \% \mathrm{CI}=-0.151 \sim-0.078)$ mediated the significant association between hardiness and depressive symptoms. (3) Across both groups, hardiness negatively predicted depression symptoms $(\beta=-0.394, P<0.001)$, and behavioral inhibition positively predicted depressive symptoms $(\beta=0.297, P<0.001)$ (Figure 1$)$.

\section{Discussions}

The military occupation involves high risk, danger, and heavy workload. Hardiness prepares the personnel to cope actively and be resilient to military stress. The current study revealed that cadets had greater levels of hardiness and behavioral activation/inhibition than soldiers. Specifically, commitment was higher in cadets than soldiers. Furthermore, this study demonstrated that, (1) across both army soldiers and military medical university cadets, hardiness and behavioral inhibition significantly predicted depressive symptoms; (2) for soldiers only, behavioral inhibition mediated the significant association between hardiness and depressive symptoms; (3) for cadets only, behavioral activation-Drive significantly predicted depressive symptoms, and hardiness operates through behavioral activation-Drive to influence depressive symptoms.

4.1. Group Comparison of Hardiness and Behavioral Activation/Inhibition. Hardiness is a generalized style of functioning that includes cognitive, emotional, and behavioral features. Therefore, high hardy individuals tend to evaluate stress as challenge rather than threat. This adaptive cognitive style of hardy individuals manifested as cognitive flexibility during conscious and automatic emotion-regulatory processes [32]. The current study observed greater levels of hardiness in cadets which may enable these individuals to be more resilient to potentially threatening experiences and reduce the risk of or maintenance of depressive symptoms. However, the depressive symptoms of cadets were comparable to army soldiers which could be explained with the differentiated mediating role of motivation (e.g., behavioral activation/inhibition) of both groups. For example, for those who with greater hardiness, commitment to specific values and goals may enable the individuals to be actively engaged in life/work. The behavioral activation may lead to increasing rewarding experience which in turn helps alleviate the depressive symptoms [33]. Additionally, the sense of control in hardy individuals may help them realize the contingency 
between effort and reward, which makes these individuals tend to increase their efforts in the short term and increase the compensatory approach motivation to overcome the frustration associated with the sense of loss of control in the long term [34]. However, for those who with less hardiness, avoidance motivation as mediator may act as adaptive coping to alleviate the detrimental effect of aversive environment stimuli and decrease the risk of depressive symptoms.

4.2. Mediation Model between Hardiness and Depressive Symptoms. First, this study confirmed the mediating rather than predictive role of approach motivation on depressive symptoms in military personnel. The approach motivation has an effect on depressive symptoms with group specificity. For the sample of military medical university cadets rather than army soldiers, behavioral activation-Drive mediated the relationship between hardiness and depressive symptoms. Sensitivity to rewards (BAS) (esp. Fun Seeking and Reward Responsiveness) plays an important role in cognitive processing (e.g., updating and working memory maintenance) of potential reward stimuli [19]. Furthermore, individuals with greater behavioral activation-Drive exhibit stronger motivation to pursue goals, regardless of whether these goals are inherently pleasurable [28]. This may enhance the opportunities to response-contingent positive reinforcement and lead to positive affect and wellbeing [35]. This result may help explain why hardiness could be enhanced through systematic training by choosing controllable goals and effective skills to deal with challenges [36,37]. Therefore, the role of behavioral activation, especially the persistent pursuit of goals, should be emphasized during hardiness training of army soldiers. However, stronger trait avoidance motivation is also associated with increased risk of onset and chronicity of depressive disorders [24], which suggested the significance of inflexible coping strategies in less hardy individuals.

Second, this study found that the avoidance motivation mediated the relationship between hardiness and depressive symptoms in army soldiers, while positively predicted depressive symptoms in military medical cadets. Moderate level of sensitivity to punishment (BIS) may predispose the depressed individuals to negative attention bias towards negative stimuli and greater approach behavior towards disliked activities $[19,38]$. In contrast, individuals with greater avoidance motivation may be more directly related to experience negative affect [35], negative cognitive/physiological reactivity [39], and depressive symptoms [40]. Meanwhile, BAS sensitivity (Drive) may work against the protective role of hardiness towards depressive symptoms. Therefore, the reduction of avoidance motivation is also an important target for evidence-based Behavioral Activation Treatment of Depression (BATD), which helps the depressed military personnel to set goals of personal value and surmount obstacles and finally to experience personal rewards after goal-attainment [41, 42]. Accordingly, training programs to increase hardiness and decrease avoidance motivation in high-stress occupations such as the military are surely needed.

Limitations. The results of the present study suggest that hardiness is an important variable contributing to depressive symptoms of soldiers and military medical cadets, with avoidance motivation as potential mediating factor. One major limitation is the cross-sectional nature of this study. Participants answered questions regarding hardiness, motivation, and depressive symptoms at the same time, which may inflate the correlations between these variables. Therefore, longitudinal study is needed to verify the main findings of this study. Although these results are suggestive regarding the underlying mediating processes through which hardiness affects depressive symptoms, the robustness of the observed relationships between hardiness, motivation, and depressive symptoms was evidenced in at least two types of regression (linear; logistic). Nonetheless, replication of these findings in separate demographically similar samples (such as Army Reserve Medical Unit and Special Forces) and higher stress situations is needed for generalization of the results. Another limitation of the study is the relatively modest sample size, although it met the standard of mediation test proposed by Fristz and MacKinnon [43]. However, future study with larger sample size is combined with advanced statistical models including stress variables to examine the stress-resilience assumption.

\section{Data Availability}

The data studied is available from the authors upon request.

\section{Conflicts of Interest}

We declare no conflicts of interest.

\section{Authors' Contributions}

Linkun Zhai, Ruicheng Wu, Tianhao Huang, Renqiang He, Yang Xiao, Yang Yu, and Xiangji Kong collected the data; Xiaoxia Wang and Linkun Zhai designed the research; Xiaoxia Wang analyzed the data and wrote the manuscript; Janet Yuen-Ha Wong provided helpful critical advice on the manuscript; Xiaoyan Zhou and Hui Yang made substantial contributions to the research design and draft of the manuscript.

\section{Acknowledgments}

This research was financially supported by the Key Project of the Applied Basic Research Programs for Military Mental Health (BWS14J029), the Youth Cultivation Foundation of Medical Science in Army Medical University (2016XPY08), the National Youth Cultivation Foundation of Military Medical Science (17QNP002), and the Chongqing Social Science Planning Project (2017QNSH21). We would like to express our gratitude to all the participants in the study. 


\section{References}

[1] P. T. P. Wong, L. C. J. Wong, and C. Scott, "Beyond Stress and Coping: The Positive Psychology of Transformation," in Handbook of Multicultural Perspectives on Stress and Coping, P. T. P. Wong and L. C. J. Wong, Eds., pp. 1-26, Springer, Boston, Mass, USA, 2006.

[2] S. R. Maddi, "Relevance of hardiness assessment and training to the military context," Military Psychology, vol. 19, no. 1, pp. 61-70, 2007.

[3] S. C. Kobasa, "Stressful life events, personality, and health: an inquiry into hardiness," Journal of Personality and Social Psychology, vol. 37, no. 1, pp. 1-11, 1979.

[4] S. R. Maddi, "On hardiness and other pathways to resilience," American Psychologist, vol. 60, no. 3, pp. 261-262, 2005.

[5] A. M. Sandvik, P. T. Bartone, S. W. Hystad, T. M. Phillips, J. F. Thayer, and B. H. Johnsen, "Psychological hardiness predicts neuroimmunological responses to stress," Psychology, Health \& Medicine, vol. 18, no. 6, pp. 705-713, 2013.

[6] A. Abdollahi, M. Abu Talib, S. N. Yaacob, and Z. Ismail, "Hardiness as a mediator between perceived stress and happiness in nurses," Journal of Psychiatric and Mental Health Nursing, vol. 21, no. 9, pp. 789-796, 2014.

[7] S. M. Escolas, B. L. Pitts, M. A. Safer, and P. T. Bartone, "The protective value of hardiness on military posttraumatic stress symptoms," Military Psychology, vol. 25, no. 2, pp. 116-123, 2017.

[8] R. Delahaij, A. W. Gaillard, and K. van Dam, "Hardiness and the response to stressful situations: Investigating mediating processes," Personality and Individual Differences, vol. 49, no. 5, pp. 386-390, 2010.

[9] P. G. Williams, D. J. Wiebe, and T. W. Smith, "Coping processes as mediators of the relationship between hardiness and health," Journal of Behavioral Medicine, vol. 15, no. 3, pp. 237-255, 1992.

[10] V. Rice, B. Liu, and L. A. Mallak, "Personal resilience and coping Part II: Identifying resilience and coping among U.S. military service members and veterans with implications for work," Work, vol. 54, no. 2, pp. 335-350, 2016.

[11] M. K. Taylor, R. Pietrobon, J. Taverniers, M. R. Leon, and B. J. Fern, "Relationships of hardiness to physical and mental health status in military men: a test of mediated effects," Journal of Behavioral Medicine, vol. 36, no. 1, pp. 1-9, 2013.

[12] V. Florian, M. Mikulincer, and O. Taubman, "Does hardiness contribute to mental health during a stressful real-life situation? The roles of appraisal and coping," Journal of Personality and Social Psychology, vol. 68, no. 4, pp. 687-695, 1995.

[13] P. T. Bartone, S. W. Hystad, J. Eid, and J. I. Brevik, "Psychological hardiness and coping style as risk/resilience factors for alcohol abuse," Journal of Archives in Military Medicine, vol. 177, no. 5, pp. 517-524, 2012.

[14] Å. G. Thomassen, S. W. Hystad, B. H. Johnsen, G. E. Johnsen, and P. T. Bartone, "The effect of hardiness on PTSD symptoms: A prospective mediational approach," Military Psychology, vol. 30, no. 2, pp. 142-151, 2018.

[15] Å. G. Thomassen, S. W. Hystad, B. H. Johnsen, G. E. Johnsen, J. C. Laberg, and J. Eid, "The combined influence of hardiness and cohesion on mental health in a military peacekeeping mission: A prospective study," Scandinavian Journal of Psychology, vol. 56, no. 5, pp. 560-566, 2015.

[16] N. E. Hundt, A. M. Williams, J. Mendelson, and R. O. NelsonGray, "Coping mediates relationships between reinforcement sensitivity and symptoms of psychopathology," Personality and Individual Differences, vol. 54, no. 6, pp. 726-731, 2013.
[17] J. A. Gray, "Implications of the neuropsychology of anxiety for personality," Clinical Neuropharmacology, vol. 7, 1984.

[18] P. J. Corr, The Reinforcement Sensitivity Theory of Personality, Cambridge University Press, New York, NY, USA, 2008.

[19] A. J. Heritage, L. J. Long, G. F. Woodman, and D. H. Zald, "Personality correlates of individual differences in the recruitment of cognitive mechanisms when rewards are at stake," Psychophysiology, vol. 55, no. 2, Article ID e12987, 2018.

[20] J. Atkinson, "Commonalities in the association of behavioral activation and behavioral inhibition with problem gambling and alcohol use in young adult college students," Journal of Gambling Studies, vol. 35, no. 1, pp. 125-141, 2019.

[21] J. Gruber, K. E. Gilbert, E. Youngstrom, J. K. Youngstrom, N. C. Feeny, and R. L. Findling, "Reward dysregulation and mood symptoms in an adolescent outpatient sample," Journal of Abnormal Child Psychology, vol. 41, no. 7, pp. 1053-1065, 2013.

[22] G. Hervas and C. Vazquez, "Low Spirits keep rewards subdued: decreases in sensitivity to reward and vulnerability to dysphoria," Behavior Therapy, vol. 44, no. 1, pp. 62-74, 2013.

[23] L. C. Quilty, L. Mackew, and R. M. Bagby, "Distinct profiles of behavioral inhibition and activation system sensitivity in unipolar vs. bipolar mood disorders," Psychiatry Research, vol. 219, no. 1, pp. 228-231, 2014.

[24] S. Y. Struijs, F. Lamers, M. Rinck, K. Roelofs, P. Spinhoven, and B. W. Penninx, "The predictive value of Approach and Avoidance tendencies on the onset and course of depression and anxiety disorders," Depression and Anxiety, vol. 35, no. 6, pp. 551-559, 2018.

[25] A. Pinto-Meza, X. Caseras, J. Soler, D. Puigdemont, V. Pérez, and R. Torrubia, "Behavioural inhibition and behavioural activation systems in current and recovered major depression participants," Personality and Individual Differences, vol. 40, no. 2, pp. 215-226, 2006.

[26] P. T. Bartone, "Test-retest reliability of the dispositional resilience scale-15, a brief hardiness scale," Psychological Reports, vol. 101, no. 3, pp. 943-944, 2016.

[27] J. Y. Wong, D. Y. Fong, A. W. Choi et al., "Transcultural and psychometric validation of the Dispositional Resilience Scale (DRS-15) in Chinese adult women," Quality of Life Research, vol. 23, no. 9, pp. 2489-2494, 2014.

[28] C. S. Carver and T. L. White, "Behavioral inhibition, behavioral activation, and affective responses to impending reward and punishment: the BIS/BAS scales," Journal of Personality and Social Psychology, vol. 67, no. 2, pp. 319-333, 1994.

[29] Y. Li, Y. Zhang, Y. Jiang et al., "The chinese version of the BIS/BAS scale: reliability and validity," Chinese Mental Health Journal, vol. 22, no. 8, pp. 613-616, 2008.

[30] E. M. Andresen, J. A. Malmgren, W. B. Carter, and D. L. Patrick, "Screening for depression in well older adults: evaluation of a short form of the CES-D (Center for Epidemiologic Studies Depression Scale)," American Journal of Preventive Medicine, vol. 10, no. 2, pp. 77-84, 1994.

[31] A. F. Hayes, PROCESS: A versatile computational tool for observed variable mediation, moderation, and conditional process modeling, 2012, http://www.afhayes.com/.

[32] P. T. Bartone, "Resilience and personality," in Biobehavioral Resilience to Stress, B. J. Lukey and V. Tepe, Eds., CRC Press, New York, NY, USA, 2008. 
[33] K. Takagaki, Y. Okamoto, R. Jinnin et al., "Mechanisms of behavioral activation for late adolescents: Positive reinforcement mediate the relationship between activation and depressive symptoms from pre-treatment to post-treatment," Journal of Affective Disorders, vol. 204, pp. 70-73, 2016.

[34] K. H. Greenaway, K. R. Storrs, M. C. Philipp, W. R. Louis, M. J. Hornsey, and K. D. Vohs, "Loss of control stimulates approach motivation," Journal of Experimental Social Psychology, vol. 56, pp. 235-241, 2015.

[35] C. C. Gill, R. T. Kane, and T. G. Mazzucchelli, "Activation, avoidance, and response-contingent positive reinforcement predict subjective wellbeing," Journal of Happiness Studies, vol. 20, no. 2, pp. 331-349, 2019.

[36] A. Bakhshizadeh, S. G. Shiroudi, and J. Khalatbari, "Effect of hardiness training on stress and post partum depression," Procedia - Social and Behavioral Sciences, vol. 84, pp. 1790-1794, 2013.

[37] P. T. Bartone, L. Eid, and S. W. Hystad, "Training hardiness for stress resilience," in Military Psychology: Concepts, Trends and Interventions, N. Maheshwari and V. V. Kumar, Eds., Sage, New Delhi, 2016.

[38] J. E. Szczepanik, M. L. Furey, A. C. Nugent, I. D. Henter, C. A. Zarate, and C. W. Lejuez, "Altered interaction with environmental reinforcers in major depressive disorder: Relationship to anhedonia," Behaviour Research and Therapy, vol. 97, pp. 170$177,2017$.

[39] E. W. Leen-Feldner, M. J. Zvolensky, M. T. Feldner, and C. Lejuez, "Behavioral inhibition: relation to negative emotion regulation and reactivity," Personality and Individual Differences, vol. 36, no. 6, pp. 1235-1247, 2004.

[40] A. R. Van Meter and E. A. Youngstrom, "A tale of two diatheses: Temperament, BIS, and BAS as risk factors for mood disorder," Journal of Affective Disorders, vol. 180, pp. 170-178, 2015.

[41] L. E. Egede, R. Acierno, R. G. Knapp et al., "Psychotherapy for depression in older veterans via telemedicine: A randomised, open-label, non-inferiority trial," The Lancet Psychiatry, vol. 2, no. 8, pp. 693-701, 2015.

[42] L. E. Egede, C. B. Frueh, L. K. Richardson et al., "Rationale and design: telepsychology service delivery for depressed elderly veterans," Trials, vol. 10, no. 1, 2009.

[43] M. S. Fritz and D. P. MacKinnon, "Required sample size to detect the mediated effect," Psychological Science, vol. 18, no. 3, pp. 233-239, 2016. 


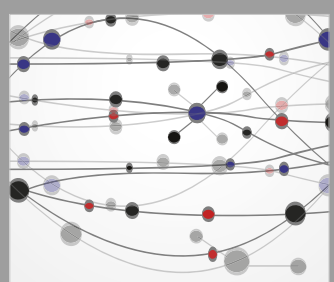

The Scientific World Journal
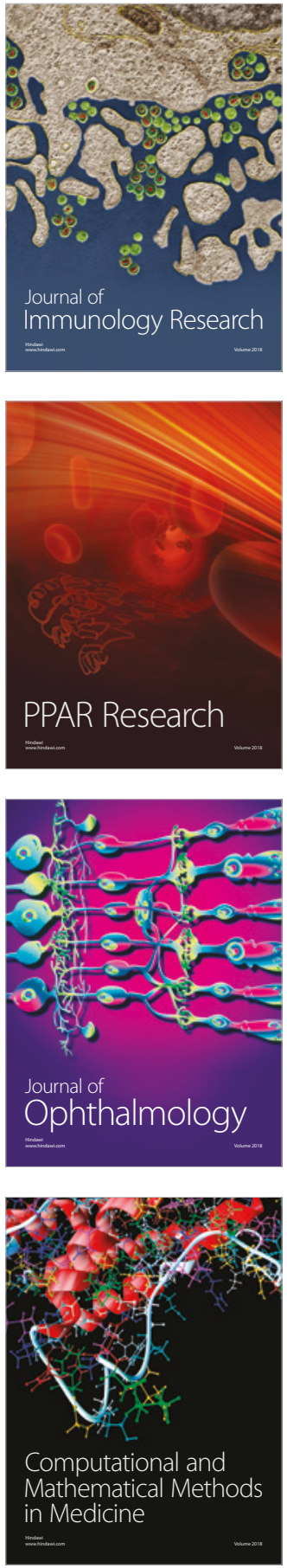

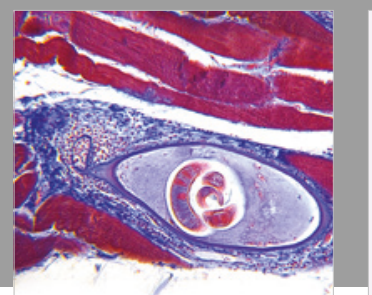

Gastroenterology Research and Practice

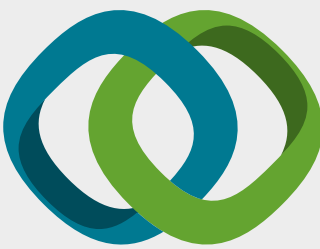

\section{Hindawi}

Submit your manuscripts at

www.hindawi.com
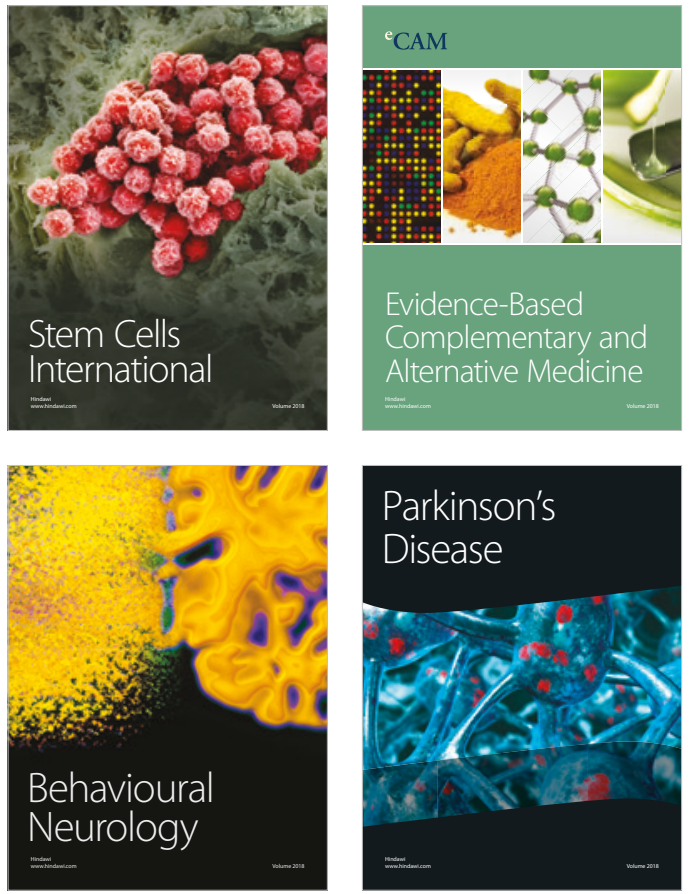

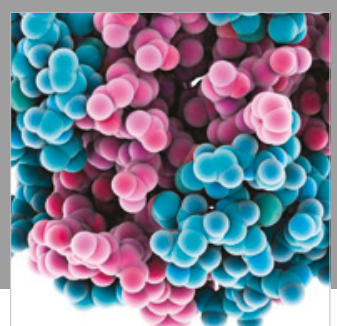

ournal of

Diabetes Research

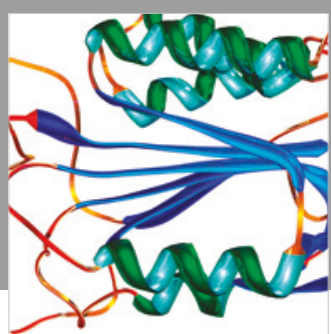

Disease Markers
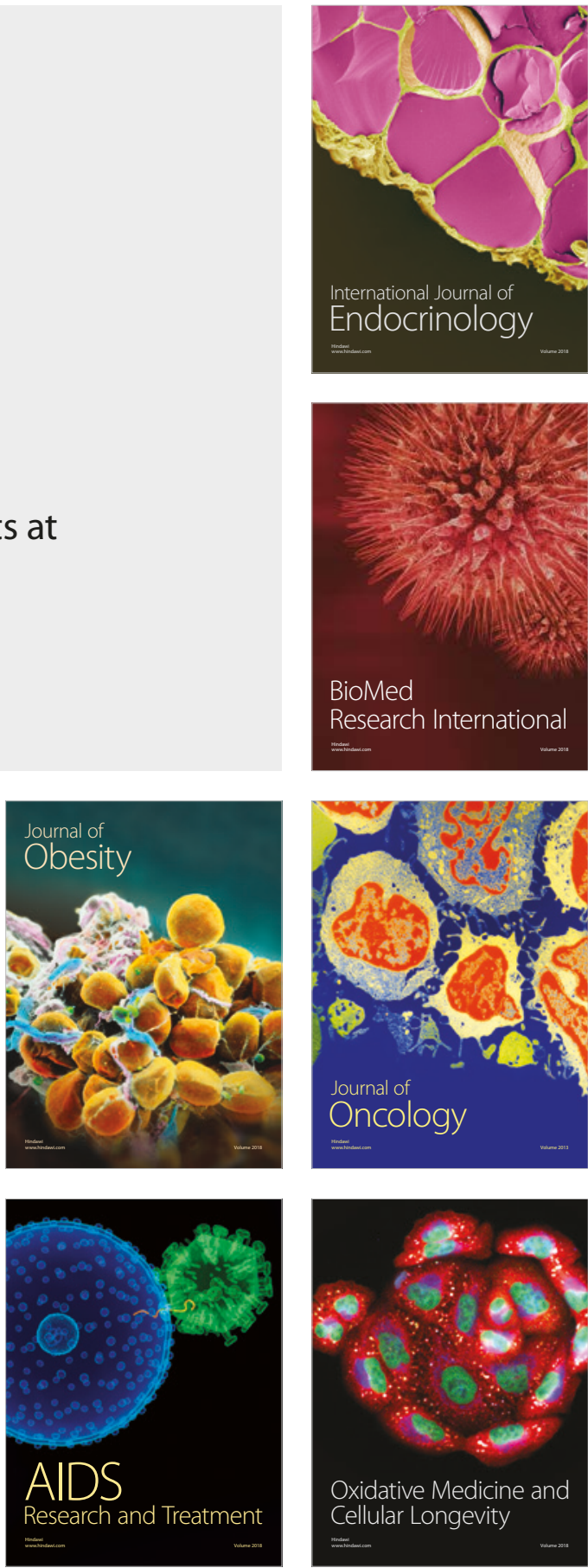